Review Article

\title{
Potential Roles of Exosomal MicroRNAs as Diagnostic Biomarkers and Therapeutic Application in Alzheimer's Disease
}

\author{
Jian-jiao Chen, ${ }^{1,2}$ Bin Zhao, ${ }^{3}$ Jie Zhao, ${ }^{1,3}$ and Shao $\mathrm{Li}^{1}$ \\ ${ }^{1}$ Department of Physiology, Liaoning Provincial Key Laboratory of Cerebral Diseases, Dalian Medical University, Dalian City, \\ Liaoning Province 116044, China \\ ${ }^{2}$ Department of General Surgery, Subei People's Hospital of Jiangsu Province, Yangzhou City, Jiangsu Province 225000, China \\ ${ }^{3}$ Technology Centre of Target-Based Nature Products for Prevention and Treatment of Aging-Related Neurodegeneration, Dalian \\ Medical University, Dalian, Liaoning, China
}

Correspondence should be addressed to Jie Zhao; dlzhaoj@163.com and Shao Li; lishao89@hotmail.com

Received 4 October 2016; Accepted 22 May 2017; Published 9 July 2017

Academic Editor: Anthony J. Hannan

Copyright (C) 2017 Jian-jiao Chen et al. This is an open access article distributed under the Creative Commons Attribution License, which permits unrestricted use, distribution, and reproduction in any medium, provided the original work is properly cited.

\begin{abstract}
Exosomes are bilipid layer-enclosed vesicles derived from endosomes and are released from neural cells. They contain a diversity of proteins, mRNAs, and microRNAs (miRNAs) that are delivered to neighboring cells and/or are transported to distant sites. miRNAs released from exosomes appear to be associated with multiple neurodegenerative conditions linking to Alzheimer's disease $(\mathrm{AD})$ which is marked by hyperphosphorylated tau proteins and accumulation of $\mathrm{A} \beta$ plaques. Exciting findings reveal that miRNAs released from exosomes modulate the expression and function of amyloid precursor proteins (APP) and tau proteins. These open up the possibility that dysfunctional exosomal miRNAs may influence AD progression. In addition, it has been confirmed that the interaction between miRNAs released by exosomes and Toll-like receptors (TLR) initiates inflammation. In exosome support-deprived neurons, exosomal miRNAs may regulate neuroplasticity to relieve neurological damage. In this review, we summarize the literature on the function of exosomal miRNAs in AD pathology, the potential of these miRNAs as diagnostic biomarkers in $\mathrm{AD}$, and the use of exosomes in the delivery of miRNAs which may lead to major advances in the field of macromolecular drug delivery.
\end{abstract}

\section{Introduction}

Alzheimer's disease $(\mathrm{AD})$ is a progressive neurodegenerative disease which is marked by aggregation of $\beta$-amyloid $(\mathrm{A} \beta)$ plaques and formation of neurofibrillary tangles (NFTs) derived from hyperphosphorylated tau proteins whose overall outcome results in neural dysfunction [1]. Majority of AD patients do not show any symptoms during the first few years (3.3 years) before the onset of dementia, at which time memory impairment might have reached its threshold [2]. Therefore, early diagnosis before the clinical phase is quite essential and could be effective for therapeutic prevention.

There are many exciting findings that have emerged in the field of extracellular vesicles during the last few years. These findings that show that application of exosomal contents which causes a change in neurological damage condition provides a bright future for the diagnosis of neurodegenerative disease [3-6]. In the central nervous system (CNS), not only neurons and astrocytes secrete exosomes but microglia and oligodendrocytes also secrete exosomes into the extracellular environment [7]. Exosomes have generated immense interest in the field of neurodegenerative disorders after their discovery as mediators delivering important proteins [8], short interfering RNA (siRNA) [9], and microRNAs (miRNAs) [3-6] in intercellular communication. Altered microRNA profiles in cerebrospinal fluid/ blood exosomes associated with neurodegenerative disorders reveal new biomarkers in the diagnosis of $\mathrm{AD}$ [3-6], and the fact that exosome is able to deliver siRNA introduces a therapeutic potential to $\mathrm{AD}[3,6,10]$.

Just like small noncoding RNAs, miRNAs have functional effects on posttranscriptional regulation of gene expression and could serve as novel diagnostic biomarkers for AD [11]. Profound works have been done to identify 
freed miRNAs in AD with anticipation of introducing reliable diagnostic biomarkers and to clarify molecular pathways that are associated with the formation of dysfunctional tau and $\mathrm{A} \beta$ aggregates [12].

Exosomal miRNAs and their pattern of transfer deserve more attention in order to establish their emerging roles and potential applications in AD. In this review, we summarize the existing knowledge of exosomal miRNAs and their involvement in $\mathrm{AD}$, emphasizing their potential to be used as diagnostic biomarkers during the preclinical phase of $\mathrm{AD}$ as well as the possibility of them being used to regulate neurodegeneration.

\section{Exosomes-Natural miRNA Carriers}

2.1. Biogenesis Pathway, Release, and Uptake of Exosomal miRNA. Exosomes are small-sized (between 30 and $100 \mathrm{~nm}$ ) spherical vesicles with an endosome-derived membrane enriched with lipids such as cholesterol, ceramide, and sphingolipids. They contain cell state-specific loads of protein, mRNA, and miRNA [13]. Some studies have shown that specific miRNAs have been involved in $\mathrm{AD}$ pathogenesis where the miRNAs bind directly to 3 '-UTR of mRNA and repress protein synthesis at the posttranscriptional level causing them to exert regulative effects [14-16]. Apart from these classical pathways (gap junction channels, apoptosis bodies, and synaptic cleft), exosomes are proposed to carry functional miRNAs coupled to the enzyme Argonaute 2 and migrate into the surrounding cells [17]. Transporting miRNAs are very secured in exosomes and retain their functional activity in target cells.

2.1.1. Formation and Secretion of Exosomes. Exosomes are derived from intraluminal vesicles of late endosomal compartments named multivesicular bodies (MVBs). It is the specific contents and membrane molecules that are contained that define exosomes irrespective of the cell types they originated from. Established exosomes contain molecules that participate in MVB biogenesis like TSG101 and Alix. They could also contain proteins involved in signal transduction such as heat shock proteins (HSP70, HSP90), certain cytoplasmic proteins such as tubulin and actin, Annexin and Rab family proteins, tetraspanins (CD9, CD63, and CD81), and major histocompatibility complex class I (MHC-I) molecules, as well as the various transmembrane proteins [7].

The fundamental step in MVB formation involves the organization of specialized domains called tetraspaninenriched membrane domains (TEMs). TEMs are enriched with membrane proteins that are necessary for vesicular fusion. In addition, TEMs also recruit some exosome membrane proteins which can be potential ligands for receptormediated internalization into the recipient cells [18]. Another specific class of membrane proteins that make up exosomes are components of endosomal sorting complex required for transport (ESCRT) machinery. These endosomal sorting complexes recognize the intraluminal vesicles which give rise to the formation of MVBs containing miRNAs. Fusion of MVBs with plasma membrane gives rise to microvesicles named exosomes which are released into the extracellular space [19]. Another important protein, Alix, is considered as a biomarker of exosome and acts together with TSG101 and CHMP4. It engages in various steps of the exosome biogenesis including cargo packaging and vesicle formation [20].

During the release of exosomes into extracellular space, any of the following could happen: (1) they may be caught by surrounding cells (or by identical cells they are derived from); (2) they may be captured by cells within distal sites; or (3) they may enter systematic circulation and be internalized into various cells [21]. Exosomes may exert its function in several distinct modes: (i) internalization into target cells and delivering their contents into these cells, (ii) attaching to the target cell's surface to trigger a second messenger signaling pathways, and (iii) releasing its contents into the extracellular space [22].

2.1.2. Biogenesis and Maturation of miRNAs. miRNAs are derived from long primary transcripts that are split by a complex of protein DGCR8/Pasha and RNAse III Drosha. These are shorter hairpin structures (50-120 nt) that constitute miRNA precursor (pre-miRNA) in the nucleus. The premiRNA is then transported into the cellular cytoplasm, converted to a short nucleotide duplex by the RNAse III enzyme Dicer, and then conveyed to miRNA-containing RNAinduced silencing complex (miRISC). In the miRISC, the single mature miRNA, which is derived from the pre-miRNA hairpin components, combines with an Argonaute protein and then migrates to specific neuronal sites [23, 24].

Alternatively, pre-miRNA and miRNA processing machinery may be collected with RNA transport granule proteins. These molecules are subsequently exported to specific neuronal sites, where either the matured or precursor miRNAs are packaged into exosomes. It must be noted that exosome as a carrier of miRNAs is novel. A schematic representation of exosomal miRNA formation is shown in Figure 1.

2.1.3. The Sorting Mechanism for Exosomal miRNAs. According to recent literature, the process of loading miRNAs into exosomes follows four main proposed ways even though the exact mechanisms has not been concluded on. Potential modes include the following: (1) the neutral sphingomyelinase 2- (nSMase2-) dependent pathway: the nSMase2 increases the quantum of exosomal miRNAs. When nSMase 2 expression and function is inhibited specifically, the quantity of exosomal miRNAs reduces, indicating the involvement of nSMase 2 in how miRNA is packaged into exosomes [25]. (2) The sumoylated heterogeneous nuclear ribonucleoprotein- (hnRNP-) dependent pathway: GGAG motif in the 3 portion of miRNA sequences can be recognized by sumoylated hnRNPA2B1, and once this recognition occurs, specific miRNAs are then packaged into exosomes [26]. (3) The 3 -end of the miRNA sequence-dependent pathway: Koppers-Lalic and his colleagues reported that the 3 -ends of uridylated endogenous miRNAs exist in exosomes that originate from B cells. The 3 -ends of an adenylated miRNA sequence are also present in B cells, indicating that the $3^{\prime}$ portion of miRNA is very vital in giving the signal to sort [27]. In addition to the 


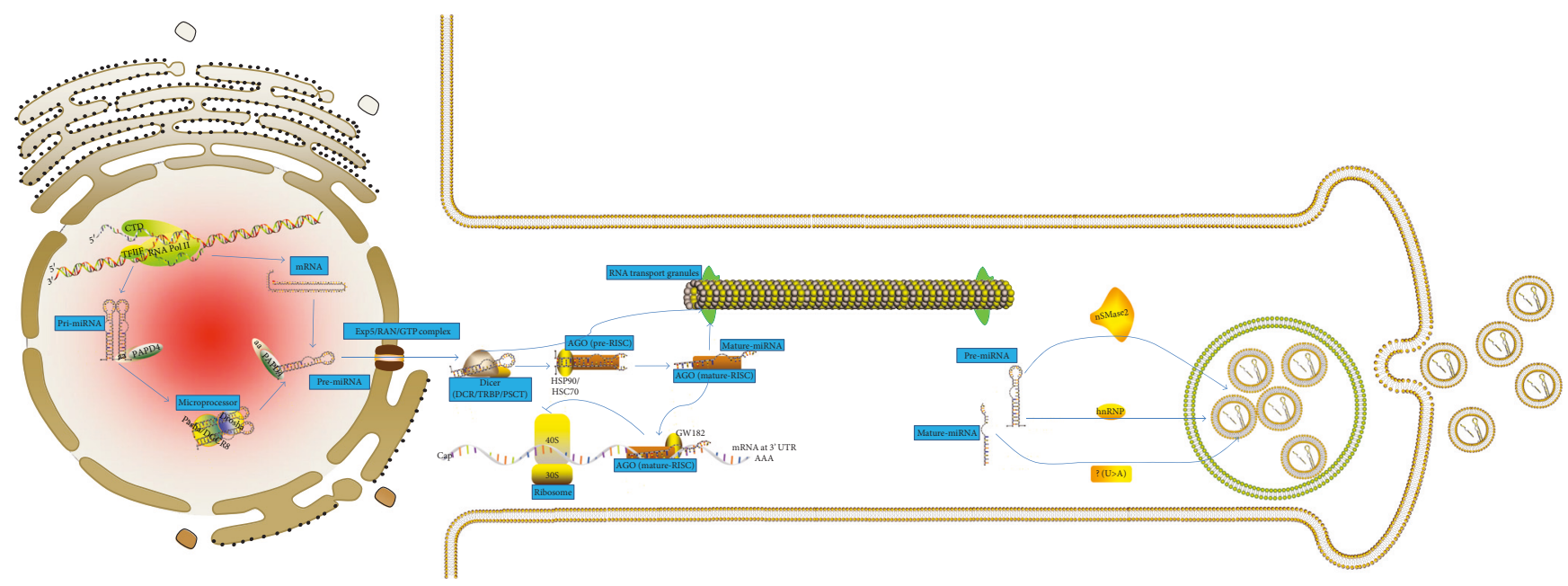

FIGURE 1: The biogenesis pathway of exosomal miRNA and composition of exosome. Canonical miRNAs are initially transcribed from long $(41 \mathrm{~kb})$ endogenous precursors called primary miRNAs that are driven by RNA pol II promoters and cleaved by microprocessor, a multiprotein complex formed by the RNase type III Drosha (the Drosophila homolog of RNASEN in humans) and the protein DGCR8 (Di George Syndrome Critical Region 8). A hairpin structure (now known as pre-miRNA) with 60-80 nucleotides in length, which bears a two-nucleotide overhang at the $3^{\prime}$ end that is a mark left by the Drosha processing, is released in this step. Within neuronal nuclei, priand pre-miRNA may be stabilized by 3 -terminal adenylation performed by PAPD4. After recognizing the precursors by their overhangs, GTPase-dependent Ran-Exp5 complex exports the pre-miRNAs out of the nucleus to the cytoplasm. An alternative pathway needs splicing out of the miRNAs from introns located in other genes, then further lariat processing, and finally proper folding into a premiRNA structure. In the cytoplasm, cleavage of the pre-miRNAs takes place once they have been loaded onto the Dicer-TRBP (TAR-RNA binding protein) complex, which removes the loop from the pre-miRNA to produce a dsRNA duplex that contains both the mature miRNA (or leader strand) and the so-called passenger strand. The Dicer-TRBP complex rapidly transfers the duplex to the miRISC (miRNA-RNAiinduced silencing complex), which contains AGO (Argonaute proteins) as its core. The HSP90/HSC70 chaperone complex participates in the process. In the miRISC, the passenger strand is degraded by an unidentified mechanism, leaving a mature miRISC loaded with a fully mature single-stranded miRNA (19-22 nt) to bind canonically to non-fully complementary mRNA targets at their $3^{\prime}$ untranslated regions (UTR). Alternatively, pri-miRNAs and miRNA may be loaded with proteins of RNA transport granules. These molecules are then transported to specific neuronal compartments, where mature or precursor miRNAs are enveloped in exosomes to be released elsewhere. Mature miRNAs are sorted into exosomes via four potential mechanisms: (1) the neutral sphingomyelinase 2- (nSMase2-) dependent pathway; (2) the miRNA motif and sumoylated heterogeneous nuclear ribonucleoprotein- (hnRNP-) dependent pathway; (3) the 3 -end of the miRNA sequence-dependent pathway; and (4) the miRNA-induced silencing complex- (miRISC-) related pathway (not shown).

above three selective modes, Guduric-Fuchs and his colleagues also revealed a possible involvement of miRISC in exosomal miRNA sorting mechanism, that is, (4) the miRNA-induced silencing complex- (miRISC-) related pathway: when $\mathrm{AGO} 2$ is knocked out, the main component of miRISC which prefers to bind to the $5^{\prime}$-end of miRNA reduces the number of privileged-transported miRNAs, such as miR451 and miR-150, in HEK293T-derived exosomes [28]. Other findings have also confirmed the relationship and involvement of miRISC with exosomal miRNA sorting mechanism [29]. Specific sequences of miRNAs may guide their loading into exosomes, while some proteins may also contribute to the sorting of exosomal miRNAs.

2.2. Localization of Exosomal miRNA in Neural Compartments. Since the CNS appears to be an abundant store of miRNA expression, it is suggested that the relevance of miRNA within the CNS is determined by neural-specific miRNA epigenetic modulative effect during brain embryogenesis [30]. Microarray analyses show how the function of miRNA regulates its function epigenetically during CNS development. This shows an important aspect of neural function especially neuroplasticity [31]. A study by Zhang et al. has indicated that specific miRNAs are profusely expressed in fetal hippocampus. In their work, the turnover in miRNA complexity occurred in $\mathrm{AD}$ hippocampus and therefore supported the hypothesis that modifiable miRNAmediated processing of mRNA group may result in neural dysfunction [32].

As a carrier and transporter of miRNA, exosomes are disseminated from various cells including neurons [33] and astrocytes [34]. In addition, circulating exosomes in neurodegenerative disorders have a distinct miRNA signature that may be involved in the pathology of these disorders $[3,4]$. It is possible that changes in miRNA expression exert an essential but uncharacteristic role in the neurodegenerative progression. There is limited evidence supporting this in the literature available; hence, the alteration in exosomal miRNA signature could be a result of the disease pathology which needs further research to clarify.

2.3. Association of Exosomal miRNAs with Neurodegeneration. Exosomes are endosome-derived membrane vesicles that carry a variety of cellular proteins, mRNA and miRNA [35]. Some findings strongly support the hypothesis that exosome-mediated miRNA signature could be utilized in the diagnosis of neurodegenerative diseases. The decrease in cerebrospinal fluid-derived exosomal microRNA-193b [3] 
and the downregulation of plasma exosomal miR-342-3p [4] could be the signatures. Exosomal miRNAs have the potential to be a risk factor like age, sex, and apolipoprotein $\varepsilon 4$ (APOE $\varepsilon 4$ ) used for predicting $\mathrm{AD}[6]$.

Corresponding to these findings, some specific exosomal miRNAs exhibit sufficient function in reducing memory decline and cognitive dysfunction. Application of low-level IFN $\gamma$ released exosomes (IFN $\gamma$-DC-Exos) that contained miR-219 exerts functional effects including increase in myelination, reduction of oxidative stress, and improvement of remyelination [36]. In addition, exosomes which originate from multipotent mesenchyme stromal cells (MSCs) transfer packaged miR-133b to astrocytes and neurons promoting neural plasticity and neurite remodeling [37]. Besides, studies by Xin et al. that investigated exosome-derived biomarkers in neuropsychiatric diseases proposed two agents, miR-497 and miR-29c, which are expressed profoundly in the prefrontal cortices of bipolar disorder and schizophrenia patients which provided an alternative view concerning the pathogenesis of these diseases [37]. Furthermore, it was studied that miRNAs encapsulated in exosomes in the cerebrospinal fluid (CSF) undergo temporal and spatial alteration during CNS embryogenesis and development and thus concluded that this could be of physiological importance [38]. Overall, miRNAs involved in the pathogenesis of $\mathrm{AD}$ deserves attention in order to explore the fluctuations in exosome expression.

\section{Epigenetic Regulation of Exosomal MicroRNAs Associated with Alzheimer's Disease}

3.1. Neuronal Intercellular Communication Involving Exosomal miRNA. Cells must communicate with each other through exchange of information and substances constantly in order to maintain physiological homeostasis. It is generally accepted that several communication modes exist in CNS. Among these, the outstanding mode is through signal activation initiated by neurotransmitters in synaptic transmission between axons and their terminals. A growing number of evidence demonstrate that exosomes may be one of several distinct mechanisms to guarantee various trades of cellular information and substance transfer [39]. The findings that exosome mediates cytosol transfer broaden the comprehension of cellular communication which account for additional physiological and pathophysiological processes within the brain. Recently, De Toro and his team figured out the diagnosis and therapeutic potential of exosomes in neurodegenerative disorders [7].

A new exciting pathway, exosome-mediated transfer of miRNA signal from neuron to astrocyte, has been characterized: excitatory amino acid transporter 2 (EAAT2, rodent analog GLT1) in perisynaptic astrocytes modulates direct miRNA-124a transfection mediated by exosome, and this leads to selective intensification of glutamate transporter1 (GLT1) protein (but not mRNA) expression in astrocytes [40].

While neurons transfer miRNA into astrocytes through neuronal exosomes and then significantly regulate protein expression in an indirect manner [40], dendritic cells may produce exosomes containing miRNA following acute lysolecithin-induced demyelination. The exosomes that are released integrate with beneficiary cells to supply practically miRNAs that increase baseline myelination and reduce oxidative stress [36]. In addition, conveyance of miR-133b boxed in exosome-enriched extracellular particles between MSCs and neural cells promotes neural plasticity. This enhances functional recovery of learning and memory in Alzheimer's disease [37]. In that regard, apart from conveying miRNAs as messengers in neural communication, exosomes may also modulate neural regeneration.

3.2. Modulation of Neurodegenerative Signaling Pathways by Exosomal miRNA. Investigation of miRNA reveals posttranscriptional modulation function as a mechanism regulated epigenetically. A considerable amount of evidence put forward by $\mathrm{Hu}$ et al. as well as Ismail et al. points out that miRNAs mediated by exosomes exert their effects in the recipient cell and may convert target gene expression [34, 41]. Mature miRNAs bind to complementary portions within the target mRNA sequence and cause them to degrade or repress the translation process. Numerous miRNAs can bind at the 3'UTR of specific genes, while identical miRNAs may have several targets; miRNA networks therefore allow the coordination of gene expression [42].

Several specific miRNAs bind to complementary sites within 3'-UTR of key genes that determine the expression of amyloid precursor protein (APP) and beta-site APPcleaving enzyme (BACE) $[43,44]$. In AD brain, extracellular $\mathrm{A} \beta$ plaques, which ultimately lead to progressive loss of neurons, are derived from processing of APP by BACE. Significantly deregulated miRNAs targeting APP like miR-193b [3], miR-101 [45], or BACE1 like miR-29c [43, 44] influence $\mathrm{A} \beta$ generation in $\mathrm{AD}$ brain. Researchers have validated computational predictions and demonstrated that miRNAs recognize specific complementary sites within $3^{\prime}$-UTR of APP/BACE1 mRNA, and they pointed out a possible involvement of miRNA in $\mathrm{AD}$ [46].

Numerous findings in transgenic $\mathrm{AD}$ mice identify that some specific miRNA expression deviates from the general levels and that deregulation of these miRNAs can contribute to the accumulation of extracellular $\mathrm{A} \beta$ plaques in sporadic $\mathrm{AD}[47,48]$. Unusual miRNA expression is supposed to be the cause for irregular proteosomal degradation of insoluble and phosphorylated tau proteins, which are mediated by Cdk5/p25 activation [49]. Genetic ablation of Dicer in adult forebrain neurons results in hyperphosphorylation of tau causing neuronal loss in the hippocampus and ultimately impairing cognitive function [50].

Potentially, apart from degrading erroneous proteins, miRNAs transferred by exosomes may also alleviate protein scarcity via rectifying functional protein translation [51]. Association of miRNAs with Ago2 protein rectifies such biological effect on misfolded proteins and renders them functional [52]. In addition to cellular miRNAs, specific virus-encoded miRNAs may also be loaded into exosomes. Epstein-Barr virus (EBV) encoding viral miRNAs may be packaged into exosomes from infected B-cells and repress 


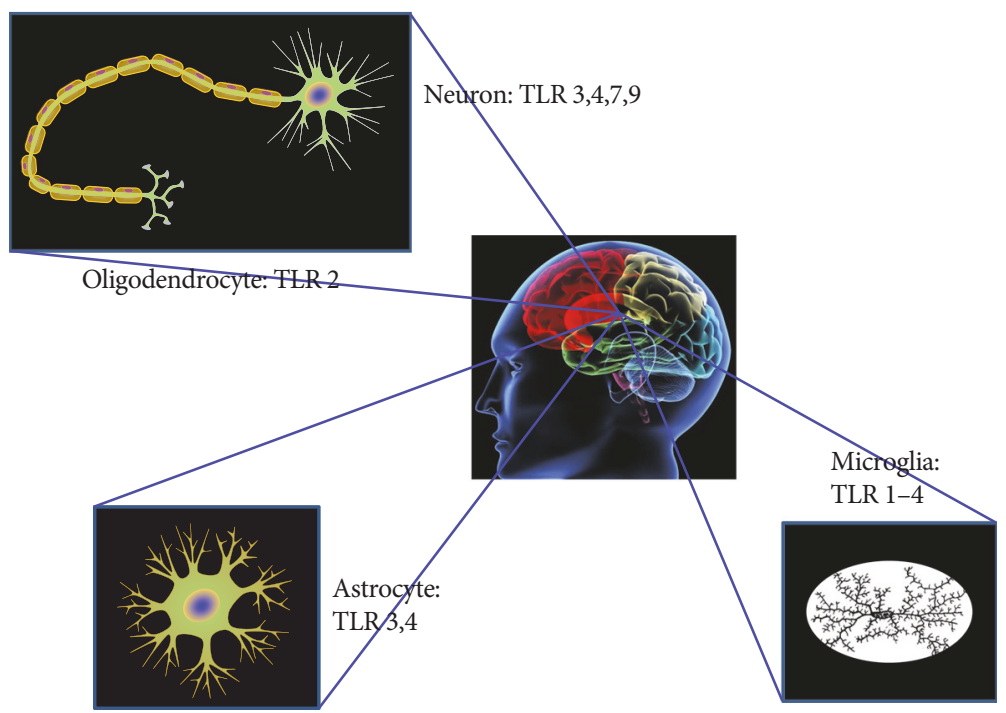

FIgURE 2: Neural cell types and their Toll-like receptor (TLR) expression. Protein profile for TLR3, 4, 7, and 9 has been reported in different neural phenotypes from humans; only TLR2 protein profile is detected in human oligodendrocytes; TLR3-4 protein accumulation is found in human astrocytes; human microglia contains TLR1-4 protein profile.

the immune-regulatory gene CXCL11 in surrounding dendritic cells derived from monocytes [53]. Besides, HIVencoded transactivation response element (TAR) miRNA could be released via exosomes and this can affect their ability to infect recipient cells [54]. All these findings broaden our knowledge and understanding about the functions of exosomal miRNAs, and this may lead us to other emerging functions.

3.3. Interplay between Exosomal miRNA and Toll-Like Receptors in Neuroinflammation. Neuroinflammation is responsible for the "double-edged sword" effect in Alzheimer's disease. Future studies that will investigate the relationship between molecules associated with neurodegeneration and receptors that initiate inflammation will bring some understanding to the mechanism of neurodegeneration [55].

It is conjectured that miRNAs mediated by exosomes may initiate Toll-like receptor (TLR) activation under certain circumstances [56, 57]. Sohrabifar et al., in their work, found that TLRs pertain to a group of pathogen-associated molecular pattern receptors and also reported that TLR polymorphisms can be related to late-onset Alzheimer disease (LOAD) susceptibility [58]. During the last decades, TLRs have been found in various cells in the brain [59-62], as shown in Figure 2. The relationship between miRNA mediated by exosomes and TLRs was deemed important in discovering the role of exosomal miRNAs in neuroinflammation of $\mathrm{AD}$.

Some findings suggest that miRNAs participate in TLRsignaling pathway at various levels, including the following: (1) targeting some components of the TLR signaling system, such as their proteins associated with signaling, regulatory molecules, and transcription factors as well as functional cytokines induced by them; (2) miRNA expression may directly be regulated by TLRs activation pathway, and (3) miRNAs may directly activate the RNA-sensing TLRs [63].
Lehmann and his group have shown that TLR7-9 recognize specific miRNAs as ligands in the CNS. For example, miRNA let-7, which is highly expressed in microglia and neurons, interacts with TLRs to regulate factors of transcription induced by TLR. Therefore, it is inferred that let-7 binds to RNA-sensing TLR7 and consequently induces neurodegeneration through neuronal TLR7 [64]. Besides, the up- and downregulation research of miR-146a brings out into the open that miR-146a may act as a potent mediator of microglial function in response to TLR2 stimulation during neurodegeneration [65]. Furthermore, in AD mouse and human brain, miR-146a localized to the hippocampal regions is full of proinflammatory cytokines in response to TLRs. These levels constitute disease severity and suggest the link between miR-146a and inflammation-induced neuropathology [66]. Zhang and his group concluded that polymorphisms in pri-miR-146a and the rare $\mathrm{C}$ allele of rs2910164 may also contribute to the genetic predisposition to $\mathrm{AD}$ by disrupting the production of miR-146a-5p which affect the expression and function of TLR2 [67]. Based on these findings, we can confirm that some specific miRNAs play a role in CNS's innate immune response and is a potential regulator modulating microglial function during neurodegeneration.

3.4. Regulatory Components of Exosomal miRNA in the Neuroplasticity Dynamics. Rapid input-restricted change in gene expression is an important aspect of neural plasticity that requires very complex mechanisms of regulating and transferring posttranscriptional mRNA. Small noncoding miRNA are positioned uniquely to support these functions by providing a nucleic acid-based specificity component for universal sequence-dependent RNA binding complexes. As a special cellular vehicle, exosomes loaded with specific miRNAs may benefit from neuroplasticity under adverse environmental conditions. 
TABLE 1: Epigenetically regulated exosomal microRNAs linked to neurodegeneration.

\begin{tabular}{lccc}
\hline MircoRNA & Role in neural network & Association with neurodegeneration & Biological fluids \\
\hline EBV-miRNA & Intercellular communication & Gene silencing & Cerebrospinal fluid \\
miRNA-124a & Intercellular communication & Neuron-to-astrocyte signaling & Cerebrospinal fluid \\
miRNA-219 & Neuronal differentiation and development & Promote CNS myelination & Cerebrospinal fluid, plasma \\
miRNA-133b & Neuronal plasticity & Benefit neurite remodeling & Cerebrospinal fluid \\
miRNA-193b & Neuronal differentiation & Affect A $\beta$ generation & Cerebrospinal fluid, plasma \\
miRNA-101 & Neuronal differentiation & Affect A $\beta$ generation & Cerebrospinal fluid \\
miRNA-29c & Neuronal differentiation & Affect A $\beta$ generation & Cerebrospinal fluid \\
miRNA-33 & Neuronal maturation and apoptosis & Downregulation influence ApoE & Cerebrospinal fluid \\
& & lipidation and A $\beta$ metabolism & Cerebrospinal fluid \\
miRNA-195 & Neuronal differentiation & Deregulate amyloid metabolism & and tau phosphorylation \\
let-7 & Neuronal inflammation & Activate the RNA-sensing TLR7 & Cerebrospinal fluid \\
miRNA-146a & Neuronal inflammation & Modulate microglial function & Cerebrospinal fluid \\
\hline
\end{tabular}

Redistribution of miRNA associated with rapid depolarization in neurons was observed by Goldie and his group and provided evidence that miRNAs exert regulatory effects on the dynamics of normal synaptic function. Intriguingly, they also found that a large proportion of depolarizationassociated changes attributed to the release of exosomes enriched with evolved miRNAs suggesting the essential vehicular role of exosome [33]. Systemic administration of exosomes that are released from mesenchymal stromal cells (MSCs) enhance neurite remodeling, neurogenesis, and angiogenesis $[68,69]$, and this effect may be a result of neural plasticity enhanced by exosomal miRNAs. Exosomes derived from MSCs transfer miR-133b to astrocytes and neurons, and this subsequently increases axonal plasticity and benefits from neurite remodeling $[37,70]$.

Gray matter injury is firmly associated with cognitive dysfunction. It was found that cognitive decline follows with neurodegeneration from myelin loss and that age-associated deficiency due to remyelination significantly contributed to AD progression [71]. Research by Pusic et al. demonstrated that environmental enrichment with serum-derived exosomes that contained miR-219 are critical for the production of myelinated oligodendrocytes, and this can be done by reducing the expression of inhibitory differentiation regulators [72]. What is more, dendritic cells (DCs) that were stimulated with IFN $\gamma$-released exosomes (IFN $\gamma$-DC-Exos) and enriched in miRNA species reduced oxidative stress increased baseline myelination and improved remyelination following acute lysolecithin-induced demyelination [36]. Interestingly, these exosomes containing specific miRNAs that had been derived from neuronal progenitor cells promoted neuronal differentiation of MSC and thus provides potential applications for tissue regeneration [73].

These findings further support the hypothesis that exosomal miRNA could be used as neural plasticity regulator. The association of the abovementioned exosomal miRNAs with neurodegeneration are summarized in Table 1. As synaptic function is thought to be dysfunctional in neurodegenerative conditions, it is plausible that exosomal
miRNA signature could be used as a potential diagnostic marker for Alzheimer's disease.

\section{Exosomal miRNAs: Novel Insights into Biomarkers for AD}

Current therapeutic activities for neurodegenerative disorders are restricted not only due to curative deficiency but also due to the limited understanding of their underlying mechanisms and also the difficulties posed in accurately diagnosing $\mathrm{AD}$ during the subclinical stages. Since pathological changes initiated years before the appearance of clinical symptoms, predictable biomarkers for the detection of $\mathrm{AD}$ are critical so much that preventative strategies could be applied to retard cognitive decline. In a neurodegenerative disorder research by Bellingham and his colleagues, a small RNA deep sequencing study demonstrated that neuronal exosomes contained a diverse range of RNA species which included retroviral RNA repeat regions, messenger RNA fragments, transfer RNA fragments, noncoding RNA, small nuclear RNA, small cytoplasmic RNA, and silencing RNA as well as known and novel candidate miRNA and that these circulating exosomes had a distinct miRNA signature that could be utilized for diagnosing neurodegenerative disorders [74].

4.1. Feasibility of Exosomal miRNAs as Biomarkers for AD. Recent development in molecular biology has prompted the potential of biomarker research from a notion of pathogenesis and specific incident that leads to a disorder originating from biosystem deterioration. Specific profiles of exosomal miRNAs from human biological fluids such as plasma and CSF have prompted the potential application of miRNAs as diagnostic biomarkers [3-6]. However, there are few obstacles that stand in the way, especially how specific miRNAs can pass through the blood-brain barrier (BBB), a mechanism that is unknown. The BBB serves as a barrier with specialized molecular machinery that mediate delivery of macromolecules, while making sure that viruses and bacteria fail to pass through it [75]. Under normal conditions, $A \beta$ is 
mediated by low-density lipoprotein protein receptor that transports it across the $\mathrm{BBB}$, suggesting the possibility of other neurodegenerative biomarkers to cross this barrier. Several presumable mechanisms by which miRNAs can cross the BBB have been put forward. In substance, it is a general consensus that some pathological conditions in neurological disorders may cause perforations of $\mathrm{BBB}$ which consequently leads to the release of cellular components [75]. In a study, the postmortem brains of late-stage $\mathrm{AD}$ patients reported that neurodegenerative conditions disrupt $\mathrm{BBB}$ vascular basement membrane [76]. Inspiringly, exosomes are proposed to function as transporters of miRNA that traverse the endothelial cellular layers of the BBB and this could facilitate communication between the brain and distant organs via biological fluids [77]. It has been proposed that the mechanism that enables exosomes to cross endothelial cells of the $\mathrm{BBB}$ can be by a receptor-mediated endocytosis [78]. In this regard, miRNAs mediated by exosomes may serve as a class of biomarkers in neurodegenerative disorders.

Alterations that occur in a biosystem often affect substances within exosomes released by neural cells, and these enlighten the possibility of exosomal miRNAs as potential biomarkers for diverse pathological states wherein deregulated miRNAs may be used for early diagnosis. In a study, exosomal miRNAs obtained from postmortem prefrontal cortices of subjects who had been diagnosed with schizophrenia and bipolar disorders were varied significantly from matched controls [79]. It was also reported that schizophrenic patients exhibited elevated levels of exosomal miR497, while elevated levels of exosomal miR-29c were also found in patients with bipolar disorder. Furthermore, researches identifying neurodegeneration-associated exosomal miRNAs in the CSF may verify the utility of exosomes to serve as biomarker carriers for neurological diseases. Furthermore, miR-193b has been shown to be present in the hippocampus of $\mathrm{AD}$ mice. It has been demonstrated that overexpression of miR-193b could suppress the expression of APP. This shows that miR-193b may get involved in the neurodegenerative process, and therefore, exosomal miR$193 \mathrm{~b}$ has the potential to be a unique biomarker for $\mathrm{AD}$ [3]. It is unlikely that a single specific miRNA could be used as $\mathrm{AD}$-associated signature. In researches looking for biomarkers that correlate with diagnosis of $\mathrm{AD}$, a 7-miRNA $\mathrm{AD}$-associated signature was obtained in a machine learning model for predicting AD status of individuals with $83-89 \%$ accuracy [4]. A similar study also selected an AD-specific 16-miRNA signature, along with established risk factors contributed to predicting AD [6]. Intriguingly, in Parkinson's disease (PD) research by Gui et al., microRNA profiling strategy for exosomal miRNAs isolated from CSF was suggested to serve for differential diagnosis of $\mathrm{PD}$ with $\mathrm{AD}$ [5]. The purpose of exosomal miRNA biomarker researches is to be able predict future cognitive decline in asymptomatic individuals and the progression of the disease in patients with early dementia.

4.2. Appropriate Biological Fluids for Detecting Exosomal miRNAs. Analysis of biological fluids for detecting brainassociated miRNAs has been focused on CSF and blood so far. As biological fluid yielded in the choroid plexus, CSF circulates through the inner ventricular system, crosses the $\mathrm{BBB}$, and is integrated into the bloodstream. On the basis of these characteristics, CSF may serve as the appropriate source containing biomarkers for $\mathrm{AD}[3,5]$. However, owing to its invasive process of sample collection, biomarkers associated with blood for AD testing may be much more widely applicable and acceptable. Identifiable miRNAs as biomarkers in blood exhibit an added advantage for early clinical diagnosis. Nevertheless, scanty researches identify miRNA as biomarkers for AD patients using blood samples. In a research that assessed the value of exosomal miRNAs as biomarkers for $\mathrm{AD}$, the $\mathrm{P} 3$ fraction of plasma, isolated by differential centrifugation and enriched in exosomes, revealed a profile of miRNA changes occurring in $\mathrm{AD}$ [4]. In another research, exosomes isolated from serum proved to be highly enriched in miRNA and an AD-specific 16-miRNA signature represented a vital step towards developing a cost-effective, noninvasive, and low-risk diagnostic application. Furthermore, it is feasible to isolate exosomes from plasma and serum by differential ultracentrifugation to profile-specific exosomal miRNAs. Generally, urine is collected noninvasively to obtain urine cellular sediment from low-speed centrifugation to analyze miRNAs implicated in prostate and bladder cancers [80]. However, analysis of urine cell sediments may not be appropriate for neurodegenerative diseases in the sense that it is enriched with cell debris that originate from the hematologic and renal epithelial as well as certain microorganisms. With appropriate samples, advances to improve sensitivity and accuracy in detecting diagnostic biomarkers would be valuable in investigating the application of exosomal miRNAs as novel biomarkers for neurodegenerative disorders.

4.3. Innovative Approach for Analysis of Exosomal miRNA. Over the last decades, new approaches to obtain large quantities of exosomes have been applied in trials including ultrafiltration, and the methods for attempting to acquire pure exosomes have been developed as utilization of monoclonal antibodies targeted membranous marker proteins of exosomes. Furthermore, exosomes have been classified according to various characteristics such as their density, morphology, size, expression of marker proteins, and intracellular origin [7].

Studies of CSF from AD patients have applied the use of miRNA microarrays, multiplex miRNA qPCR assay, or target candidate miRNA approaches to identify differentially expressed miRNA [81]. Recently, the progress in deepsequencing technology has presented a realizable approach to detect deregulated exosomal miRNAs in neurodegenerative diseases $[4,6]$. Deep-sequencing method permits exhaustive analyses of numerous sequence data, initiating revolutionary accelerated research compared to traditional methods. This has shown to be an effective approach to identifying accurate encoded information from nucleotide fragments [82]. Benefits of deep sequencing rely on their capability to be simultaneously operated on a large quantity of independent sequencing events. Due to the sensitivity and accuracy of deep sequencing, it is able to identify genetic 
modifications, particularly deregulated miRNA, in neurodegenerative disease [82].

\section{Synthetic miRNA Delivered by Exosomes: A Potential Therapy for AD}

Previous reports have demonstrated that dysfunction of miRNAs is involved in the pathogenesis of human $\mathrm{AD}$ and that the therapeutic potential of miRNAs could guide us towards the development of new therapeutic strategies in neurodegenerative disorder. In a study, upregulating miR188-3p in the hippocampus suppressed BACE1 expression and $\mathrm{A} \beta$ formation and this prevented deteriorations in long-term potentiation, spatial learning, and memory in the hippocampus [32]. It is unlikely that miR-188-3p is unique in its ability to regulate $\mathrm{A} \beta$ formation, but the role of miR$188-3 p$ is of critical interest, given that its recognition element in the BACE1 $3^{\prime}$-UTR is extraordinarily conserved. Apart from the accumulation of $\mathrm{A} \beta$, another neuropathological hallmark of $\mathrm{AD}$, neurofibrillary tangles (NFTs) result from tauopathy which may also be regulated by miRNA as well. For example, miR-219 binds directly to the 3'-UTR of the tau mRNA and represses tau synthesis at the posttranscriptional level. Modification of the silencing mechanism of tau by miR-219 that is perturbed during neurofibrillary degeneration suggested that developing therapies for tauopathies is possible [14]. In this case, favorable natural transport of nanovesicles represent another major barrier to avoid or alleviate immunogenicity of the miRNA or its delivery vehicle, especially if repeated doses are needed to treat degenerative diseases.

Recent researches have revealed that exosomes mediate horizontal delivery of distinct RNAs in intercellular communication. The utilization of exosomes for transport of certain therapeutic nucleic acids to recipient cells is considered as a potential approach for $\mathrm{AD}$ [51]. Involvement of exosomal miRNAs with pathogenesis of neurodegenerative disorders urges researchers to investigate their therapeutic potential too.

The capacity of exosomes to deliver functional miRNA pushes the hope of replacing the already known virus-based gene therapy with this [83]. When this is compared to current RNA interference (RNAi) approaches which includes utilization of viral and synthetic delivery systems such as liposomes and endogenous exosomes, the body's own intercellular carrier may internalize into recipient cells without evoking any immune responses. This therefore assures that an advancement in the field of drug delivery is near possibility [7].

Exosomes serve as not only vehicles to deliver miRNA but also synthetic nucleic acids, for instance, short interfering RNA (siRNA) which are therapeutic compounds and have been exploited recently. Researchers obtained exosomes from self-derived dendritic cells and then decorated exosomes to express membrane protein Lamp2b and a neuron-specific RVG peptide in order to deliver cargoes specifically to recipient neural cells. Intravenously injected RVG-targeted exosomes delivered GAPDH siRNA which resulted in knockdown of BACE1 and mediated the formation of the peptide that forms $\beta$-amyloid plaque associated with $\mathrm{AD}$ pathogenesis [9]. These findings lighten the therapeutic potential of the application of exosome-mediated short interfering RNA approach in AD. Schematic representation of production, harvest, and readministration of targeted selfexosomes for gene delivery is illustrated in Figure 3.

By all indications, exosomes have multiple advantages over existing miRNA delivery vehicles. Since they are derived from the patients' own cells, they would be less immunogenic than other foreign delivery vehicles. Exosomes remain relatively stable in the blood as they avoid coagulation factors and complement which are most likely due to their surface expression of CD46 and CD59 [84], as well as antibody responses, due to their self-derived nature. Dendritic cellderived exosomes express membranous tetraspanin CD9, which facilitates direct fusion with recipient cells and deliver their content directly into the cytosol [85]. This entry mode bypasses the endosomal-lysosomal pathway, where the immune system recognizes nucleic acids by TLR7 and TLR8. It also circumvents the need for endosomal-escape strategies with their associated toxicities. Finally, the small size of exosomes would also be beneficial with facilitation as they pass through vessel fenestrations.

Despite these advantages, numerous issues are still being addressed before clinical implementation of this strategy. Firstly, as to whether the RVG-Lamp2b fusion-protein induces adaptive immunity after repeated administration needs to be verified. In fact, adaptive immune responses would be anticipated. Another issue not yet addressed is that the receptor coupled with RVG expressed in the surface of exosomes, alpha-7-subunit acetylcholine receptor, markedly declines in brains affected by $\mathrm{AD}$ [10]. This issue is critical when aiming to develop RVG exosomes for clinical use in AD. In addition, a stable exosome source is yet to be exploited in order to expand well characterized exosomes. The loading process of miRNAs into exosomes also needs to be optimized to achieve maximum efficiency. Last but not the least, specific targeting function of exosomes should also be further studied to ensure safe and precise delivery to recipient tissues.

With this progress in intercellular transfer researches, exosomes provide a breakthrough in the field of drug delivery, bringing an alternative source of clinical therapy.

\section{Perspectives and Conclusions}

The ability of exosome-transporting miRNAs to function in neighboring or distant cells exhibits a novel insight into modification mechanisms employed in $\mathrm{AD}$. Besides, our review also reveals gaps in knowledge that require further research.

As novel epigenetic-related systems ensure cellular information exchange, the exhaustive mechanism that underlies uptake of exosomes containing miRNAs into neighboring or distant cells needs to be explored and this holds enormous potential for RNA interference applications. In addition, investigating the ability of exosome-mediated miRNAs to bind to TLRs in the endosomes of recipient cells is still in its infancy. Vartanian and his team hypothesized that intracellular delivery of miRNAs via exosomes brings about TLR 


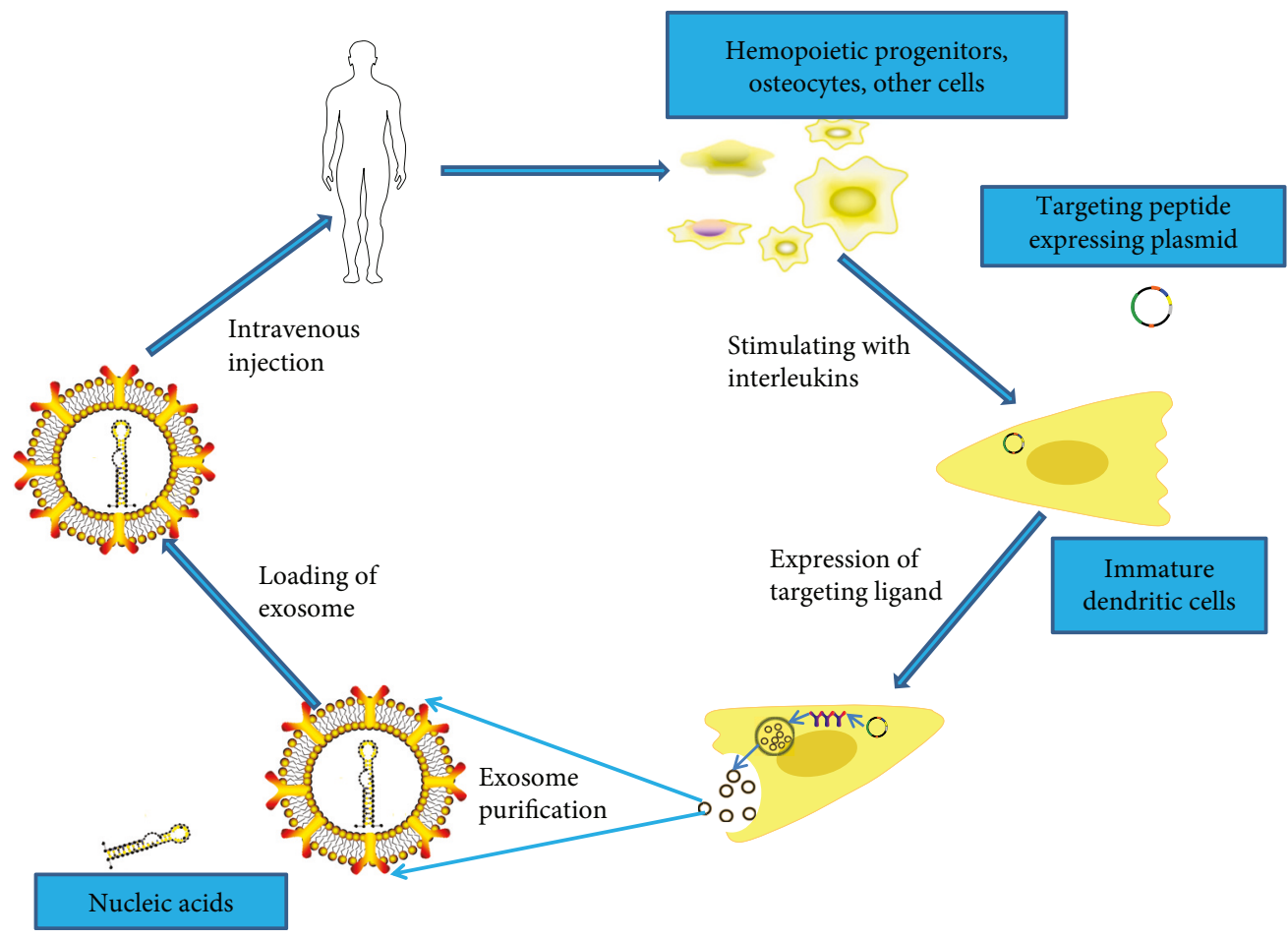

FIGURE 3: Schematic representation of production, harvest, and readministration of engineering modified exosomes for gene delivery. To acquire enough immunologically inert exosomes, harvest cells like hemopoietic progenitors and osteocytes are used as the source cell. As immature dendritic cells produce a lot of exosomes devoid of T-cell activators such as MHC-II and CD86, it could be selected as the source cell. Targeting peptides expressing plasmids (e.g., RVG (rabies virus glycoprotein)) were transfected into the source cells to get exosomes with the ability of specifically binding to neural cells. Therapeutic nucleic acids can be introduced into the modified exosomes by electroporation method. Following intravenous injection, exosomes encapsulating nucleic acids could be found within the central nervous system.

tolerance, which is a hyporesponsive state of TLR contributing to neuroprotection [86]. If this is the case, it reveals an exciting new insight into the regulation of TLRs within inflammation of $\mathrm{AD}$ and even in other CNS inflammationrelated diseases. Overall, these compelling evidences that we summarize in this review point out a complex interplay between neurodegeneration and exosomal miRNAs and these support the functional effects of exosome-containing miRNAs in neurodegenerative disorders.

Although previous researches have demonstrated that a panel of miRNAs within exosomes from the CSF and plasma may serve as fingerprints of early stages in $\operatorname{AD}[3,4,6]$, further research data is still critical to identify the function of the miRNA in order to strengthen such findings. The diagnostic validation of targets (mRNA and protein) need to be coupled with miRNAs; therefore, one of the major challenges would be the comparison of miRNA analysis with transcriptions and proteomic studies. Besides, technological aspects possessing both considerable production and meticulous analysis represent another challenge. With the need to further understand miRNA function in specific cellular events, consequent technological advances in sensitive and accurate miRNA analysis are necessary for offering prospective role for exosomal miRNAs to be used as potential diagnostic biomarkers of $\mathrm{AD}$.

A study that demonstrates an efficacious way of delivering functional siRNA into $\mathrm{AD}$ mouse brain by systemic exosome administration represents a promising approach for RNA interference applications $[9,10]$. However, we have to realize that there is still a long way ahead, and numerous hurdles need to be crossed. First of all, since the possibility of potential side effects on recipient cells has not been looked at, the safety of exosomes should never be ignored. Moreover, the precise mechanisms that underlie the processes of the blood-brain barrier cross, target cells selection, and content release have not been proven clearly, and this necessitates further investigation. Further researches are needed to establish if exosomal miRNAs could be utilized as diagnostic biomarkers of $\mathrm{AD}$ and practical RNA interference applications.

This review has summarized compelling evidence for multiple ways in which exosomal miRNAs contribute to important biological functions related to intercellular communication, neuroinflammation, and neuroplasticity. We put forward new applications of exosomal miRNAs as diagnostic biomarkers and potential gene therapy. We expect that our review will contribute to better comprehension of the roles that exosomal miRNAs play in pathological mechanisms of $\mathrm{AD}$.

\section{Conflicts of Interest}

The authors declare that they have no conflicts of interest. 


\section{Acknowledgments}

The work was supported by the National Natural Science Foundation of China (81571061, 81671061, 81371223, and 81371437).

\section{References}

[1] D. A. Bennett, L. Yu, J. Yang, G. P. Srivastava, C. Aubin, and P. L. De Jager, "Epigenomics of Alzheimer's disease," Translational Research, vol. 165, no. 1, pp. 200-220, 2015.

[2] V. L. Villemagne, S. Burnham, P. Bourgeat et al., "Amyloid beta deposition, neurodegeneration, and cognitive decline in sporadic Alzheimer's disease: a prospective cohort study," Lancet Neurology, vol. 12, no. 4, pp. 357-367, 2013.

[3] C. G. Liu, J. Song, Y. Q. Zhang, and P. C. Wang, "MicroRNA$193 \mathrm{~b}$ is a regulator of amyloid precursor protein in the blood and cerebrospinal fluid derived exosomal microRNA-193b is a biomarker of Alzheimer's disease," Molecular Medicine Reports, vol. 10, no. 5, pp. 2395-2400, 2014.

[4] G. Lugli, A. M. Cohen, D. A. Bennett et al., "Plasma exosomal miRNAs in persons with and without Alzheimer disease: altered expression and prospects for biomarkers," PloS One, vol. 10, no. 10, article e0139233, 2015.

[5] Y. Gui, H. Liu, L. Zhang, W. Lv, and X. Hu, "Altered microRNA profiles in cerebrospinal fluid exosome in Parkinson disease and Alzheimer disease," Oncotarget, vol. 6, no. 35, pp. 37043-37053, 2015.

[6] L. Cheng, J. D. Doecke, R. A. Sharples et al., "Prognostic serum miRNA biomarkers associated with Alzheimer's disease shows concordance with neuropsychological and neuroimaging assessment," Molecular Psychiatry, vol. 20, no. 10, pp. 11881196, 2015.

[7] J. De Toro, L. Herschlik, C. Waldner, and C. Mongini, "Emerging roles of exosomes in normal and pathological conditions: new insights for diagnosis and therapeutic applications," Frontiers in Immunology, vol. 6, p. 203, 2015.

[8] K. Yuyama, H. Sun, S. Usuki et al., "A potential function for neuronal exosomes: sequestering intracerebral amyloid-beta peptide," FEBS Letters, vol. 589, no. 1, pp. 84-88, 2015.

[9] L. Alvarez-Erviti, H. Yin, C. Betts, S. Lakhal, M. J. Wood, and Y. Seow, "Delivery of siRNA to the mouse brain by systemic injection of targeted exosomes," Nature Biotechnology, vol. 29, no. 4, pp. 341-345, 2011.

[10] J. G. van den Boorn, M. Schlee, C. Coch, and G. Hartmann, "SiRNA delivery with exosome nanoparticles," Nature Biotechnology, vol. 29, no. 4, pp. 325-326, 2011.

[11] H. Dong, J. Li, L. Huang et al., "Serum microRNA profiles serve as novel biomarkers for the diagnosis of Alzheimer's disease," Disease Markers, vol. 2015, Article ID 625659, 11 pages, 2015.

[12] P. Lau, C. S. Frigerio, and B. De Strooper, "Variance in the identification of microRNAs deregulated in Alzheimer's disease and possible role of lincRNAs in the pathology: the need of larger datasets," Ageing Research Reviews, vol. 17, pp. 43$53,2014$.

[13] J. Zhang, S. Li, L. Li et al., "Exosome and exosomal microRNA: trafficking, sorting, and function," Genomics, Proteomics \& Bioinformatics, vol. 13, no. 1, pp. 17-24, 2015.

[14] I. Santa-Maria, M. E. Alaniz, N. Renwick et al., "Dysregulation of microRNA-219 promotes neurodegeneration through post- transcriptional regulation of tau," The Journal of Clinical Investigation, vol. 125, no. 2, pp. 681-686, 2015.

[15] Z. Liu, C. Wang, X. Wang, and S. Xu, “Therapeutic effects of transplantation of as-MiR-937-expressing mesenchymal stem cells in murine model of Alzheimer's disease," Cellular Physiology and Biochemistry, vol. 37, no. 1, pp. 321-330, 2015.

[16] Y. Zong, P. Yu, H. Cheng et al., "miR-29c regulates NAV3 protein expression in a transgenic mouse model of Alzheimer's disease," Brain Research, vol. 1624, pp. 95-102, 2015.

[17] G. S. Higa, E. de Sousa, L. T. Walter, E. R. Kinjo, R. R. Resende, and A. H. Kihara, "MicroRNAs in neuronal communication," Molecular Neurobiology, vol. 49, no. 3, pp. 1309-1326, 2014.

[18] Z. Andreu and M. Yanez-Mo, "Tetraspanins in extracellular vesicle formation and function," Frontiers in Immunology, vol. 5, p. 442, 2014.

[19] M. Colombo, C. Moita, G. van Niel et al., "Analysis of ESCRT functions in exosome biogenesis, composition and secretion highlights the heterogeneity of extracellular vesicles," Journal of Cell Science, vol. 126, Part 24, pp. 5553-5565, 2013.

[20] B. Roucourt, S. Meeussen, J. Bao, P. Zimmermann, and G. David, "Heparanase activates the syndecan-syntenin-ALIX exosome pathway," Cell Research, vol. 25, no. 4, pp. 412-428, 2015.

[21] S. Pant, H. Hilton, and M. E. Burczynski, "The multifaceted exosome: biogenesis, role in normal and aberrant cellular function, and frontiers for pharmacological and biomarker opportunities," Biochemical Pharmacology, vol. 83, no. 11, pp. 1484-1494, 2012.

[22] C. Fruhbeis, D. Frohlich, and E. M. Kramer-Albers, "Emerging roles of exosomes in neuron-glia communication," Frontiers in Physiology, vol. 3, p. 119, 2012.

[23] J. Winter, S. Jung, S. Keller, R. I. Gregory, and S. Diederichs, "Many roads to maturity: microRNA biogenesis pathways and their regulation," Nature Cell Biology, vol. 11, no. 3, pp. 228-234, 2009.

[24] D. J. Gibbings, C. Ciaudo, M. Erhardt, and O. Voinnet, "Multivesicular bodies associate with components of miRNA effector complexes and modulate miRNA activity," Nature Cell Biology, vol. 11, no. 9, pp. 1143-9, 2009.

[25] N. Kosaka, H. Iguchi, K. Hagiwara, Y. Yoshioka, F. Takeshita, and T. Ochiya, "Neutral sphingomyelinase 2 (nSMase2)dependent exosomal transfer of angiogenic microRNAs regulate cancer cell metastasis," The Journal of Biological Chemistry, vol. 288, no. 15, pp. 10849-10859, 2013.

[26] C. Villarroya-Beltri, C. Gutiérrez-Vázquez, F. Sánchez-Cabo et al., "Sumoylated hnRNPA2B1 controls the sorting of miRNAs into exosomes through binding to specific motifs," Nature Communications, vol. 4, p. 2980, 2013.

[27] D. Koppers-Lalic, M. Hackenberg, I. V. Bijnsdorp et al., "Nontemplated nucleotide additions distinguish the small RNA composition in cells from exosomes," Cell Reports, vol. 8, no. 6, pp. 1649-1658, 2014.

[28] J. Guduric-Fuchs, A. O'Connor, B. Camp, C. L. O'Neill, R. J. Medina, and D. A. Simpson, "Selective extracellular vesiclemediated export of an overlapping set of microRNAs from multiple cell types," BMC Genomics, vol. 13, p. 357, 2012.

[29] M. Aqil, A. R. Naqvi, A. S. Bano, and S. Jameel, "The HIV-1 Nef protein binds argonaute- 2 and functions as a viral suppressor of RNA interference," PloS One, vol. 8, no. 9, article e74472, 2013. 
[30] R. Weng and S. M. Cohen, "Control of drosophila type I and type II central brain neuroblast proliferation by bantam microRNA,” Development, vol. 142, no. 21, pp. 3713-3720, 2015.

[31] A. Aksoy-Aksel, F. Zampa, and G. Schratt, "MicroRNAs and synaptic plasticity-a mutual relationship," Philosophical Transactions of the Royal Society of London. Series B, Biological Sciences, vol. 369, p. 1652, 2014.

[32] J. Zhang, M. Hu, and Z. Teng, "Synaptic and cognitive improvements by inhibition of 2-AG metabolism are through upregulation of microRNA-188-3p in a mouse model of Alzheimer's disease," The Journal of Neuroscience, vol. 34, no. 45, pp. 14919-14933, 2014.

[33] B. J. Goldie, M. D. Dun, M. Lin et al., "Activity-associated miRNA are packaged in Maplb-enriched exosomes released from depolarized neurons," Nucleic Acids Research, vol. 42, no. 14, pp. 9195-9208, 2014.

[34] G. Hu, H. Yao, A. D. Chaudhuri et al., "Exosome-mediated shuttling of microRNA-29 regulates HIV tat and morphinemediated neuronal dysfunction," Cell Death \& Disease, vol. 3, p. e381, 2012.

[35] S. Keerthikumar, D. Chisanga, D. Ariyaratne et al., "ExoCarta: a web-based compendium of exosomal cargo," Journal of Molecular Biology, vol. 428, no. 4, pp. 688-692, 2016.

[36] A. D. Pusic, K. M. Pusic, B. L. Clayton, and R. P. Kraig, "IFNgamma-stimulated dendritic cell exosomes as a potential therapeutic for remyelination," Journal of Neuroimmunology, vol. 266, no. 1-2, pp. 12-23, 2014.

[37] H. Xin, Y. Li, Z. Liu et al., "MiR-133b promotes neural plasticity and functional recovery after treatment of stroke with multipotent mesenchymal stromal cells in rats via transfer of exosome-enriched extracellular particles," Stem Cells, vol. 31, no. 12, pp. 2737-2746, 2013.

[38] A. Tietje, K. N. Maron, Y. Wei, and D. M. Feliciano, "Cerebrospinal fluid extracellular vesicles undergo age dependent declines and contain known and novel non-coding RNAs," PloS One, vol. 9, no. 11, article e113116, 2014.

[39] D. O. Borroto-Escuela, L. F. Agnati, K. Bechter, A. Jansson, A. O. Tarakanov, and K. Fuxe, "The role of transmitter diffusion and flow versus extracellular vesicles in volume transmission in the brain neural-glial networks," Philosophical Transactions of the Royal Society of London. Series B, Biological Sciences, vol. 370, p. 1672, 2015.

[40] L. Morel, M. Regan, H. Higashimori et al., "Neuronal exosomal miRNA-dependent translational regulation of astroglial glutamate transporter GLT1," The Journal of Biological Chemistry, vol. 288, no. 10, pp. 7105-7116, 2013.

[41] N. Ismail, Y. Wang, D. Dakhlallah et al., "Macrophage microvesicles induce macrophage differentiation and miR-223 transfer," Blood, vol. 121, no. 6, pp. 984-995, 2013.

[42] G. C. Shukla, J. Singh, and S. Barik, "MicroRNAs: processing, maturation, target recognition and regulatory functions," Molecular and Cellular Pharmacology, vol. 3, no. 3, pp. 8392, 2011.

[43] X. Lei, L. Lei, Z. Zhang, Z. Zhang, and Y. Cheng, "Downregulated miR-29c correlates with increased BACE1 expression in sporadic Alzheimer's disease," International Journal of Clinical and Experimental Pathology, vol. 8, no. 2, pp. 1565-1574, 2015.

[44] G. Yang, Y. Song, X. Zhou et al., "MicroRNA-29c targets betasite amyloid precursor protein-cleaving enzyme 1 and has a neuroprotective role in vitro and in vivo," Molecular Medicine Reports, vol. 12, no. 2, pp. 3081-3088, 2015.
[45] J. M. Long and D. K. Lahiri, "MicroRNA-101 downregulates Alzheimer's amyloid-beta precursor protein levels in human cell cultures and is differentially expressed," Biochemical and Biophysical Research Communications, vol. 404, no. 4, pp. 889-895, 2011.

[46] J. Denk, K. Boelmans, C. Siegismund, D. Lassner, S. Arlt, and H. Jahn, "MicroRNA profiling of CSF reveals potential biomarkers to detect Alzheimer's disease," PloS One, vol. 10, no. 5, article e0126423, 2015.

[47] J. Kim, H. Yoon, T. Horie et al., "microRNA-33 regulates ApoE lipidation and amyloid-beta metabolism in the brain," The Journal of Neuroscience, vol. 35, no. 44, pp. 14717-14726, 2015.

[48] F. Jaouen and E. Gascon, "Understanding the role of miR-33 in brain lipid metabolism: implications for Alzheimer's disease," The Journal of Neuroscience, vol. 36, no. 9, pp. 2558-2560, 2016.

[49] L. H. Sun, T. Ban, C. D. Liu et al., "Activation of Cdk5/p25 and tau phosphorylation following chronic brain hypoperfusion in rats involves microRNA-195 down-regulation," Journal of Neurochemistry, vol. 134, no. 6, pp. 1139-1151, 2015.

[50] S. S. Hebert, A. S. Papadopoulou, P. Smith et al., "Genetic ablation of Dicer in adult forebrain neurons results in abnormal tau hyperphosphorylation and neurodegeneration," Human Molecular Genetics, vol. 19, no. 20, pp. 3959-3969, 2010.

[51] R. Liu, J. Liu, X. Ji, and Y. Liu, "Synthetic nucleic acids delivered by exosomes: a potential therapeutic for generelated metabolic brain diseases," Metabolic Brain Disease, vol. 28, no. 4, pp. 551-562, 2013.

[52] L. Li, D. Zhu, L. Huang et al., "Argonaute 2 complexes selectively protect the circulating microRNAs in cell-secreted microvesicles," PloS One, vol. 7, no. 10, article e46957, 2012.

[53] D. M. Pegtel, K. Cosmopoulos, D. A. Thorley-Lawson et al., "Functional delivery of viral miRNAs via exosomes," Proceedings of the National Academy of Sciences of the United States of America, vol. 107, no. 14, pp. 6328-6333, 2010.

[54] A. Narayanan, S. Iordanskiy, R. Das et al., "Exosomes derived from HIV-1-infected cells contain trans-activation response element RNA," The Journal of Biological Chemistry, vol. 288, no. 27, pp. 20014-20033, 2013.

[55] F. L. Heppner, R. M. Ransohoff, and B. Becher, "Immune attack: the role of inflammation in Alzheimer disease," Nature Reviews. Neuroscience, vol. 16, no. 6, pp. 358-372, 2015.

[56] D. G. Phinney, M. Di Giuseppe, J. Njah et al., "Mesenchymal stem cells use extracellular vesicles to outsource mitophagy and shuttle microRNAs," Nature Communications, vol. 6, p. 8472, 2015.

[57] K. Bryniarski, W. Ptak, E. Martin et al., "Free extracellular miRNA functionally targets cells by transfecting exosomes from their companion cells," PloS One, vol. 10, no. 4, article e0122991, 2015.

[58] N. Sohrabifar, J. Gharesouran, M. Talebi, M. Ghojazadeh, and S. M. Mohaddes Ardebili, "Association of CLU and TLR2 gene polymorphisms with late-onset Alzheimer disease in a northwestern Iranian population," Turkish Journal of Medical Sciences, vol. 45, no. 5, pp. 1082-1086, 2015.

[59] M. Bsibsi, A. Nomden, J. M. van Noort, and W. Baron, "Toll-like receptors 2 and 3 agonists differentially affect oligodendrocyte survival, differentiation, and myelin membrane formation," Journal of Neuroscience Research, vol. 90, no. 2, pp. 388-398, 2012. 
[60] D. V. Chistyakov, S. E. Aleshin, A. A. Astakhova, M. G. Sergeeva, and G. Reiser, "Regulation of peroxisome proliferator-activated receptors (PPAR) alpha and -gamma of rat brain astrocytes in the course of activation by toll-like receptor agonists," Journal of Neurochemistry, vol. 134, no. 1, pp. 113-124, 2015.

[61] L. Facci, M. Barbierato, C. Marinelli, C. Argentini, S. D. Skaper, and P. Giusti, "Toll-like receptors $2,-3$ and -4 prime microglia but not astrocytes across central nervous system regions for ATP-dependent interleukin-1beta release," Scientific Reports, vol. 4, p. 6824, 2014.

[62] J. Qi, K. Buzas, H. Fan et al., "Painful pathways induced by TLR stimulation of dorsal root ganglion neurons," Journal of Immunology, vol. 186, no. 11, pp. 6417-6426, 2011.

[63] X. He, Z. Jing, and G. Cheng, "MicroRNAs: new regulators of Toll-like receptor signalling pathways," BioMed Research International, vol. 2014, Article ID 945169, 14 pages, 2014.

[64] S. M. Lehmann, C. Krüger, B. Park et al., “An unconventional role for miRNA: let-7 activates Toll-like receptor 7 and causes neurodegeneration," Nature Neuroscience, vol. 15, no. 6, pp. 827-835, 2012.

[65] R. Saba, S. Gushue, R. L. Huzarewich et al., "MicroRNA 146a (miR-146a) is over-expressed during prion disease and modulates the innate immune response and the microglial activation state," PloS One, vol. 7, no. 2, article e30832, 2012.

[66] W. J. Lukiw, P. Dua, A. I. Pogue, C. Eicken, and J. M. Hill, "Upregulation of micro RNA-146a (miRNA-146a), a marker for inflammatory neurodegeneration, in sporadic CreutzfeldtJakob disease (sCJD) and Gerstmann-Straussler-Scheinker (GSS) syndrome," Journal of Toxicology and Environmental Health. Part a, vol. 74, no. 22-24, pp. 1460-1468, 2011.

[67] B. Zhang, A. Wang, C. Xia, Q. Lin, and C. Chen, "A single nucleotide polymorphism in primary-microRNA-146a reduces the expression of mature microRNA-146a in patients with Alzheimer's disease and is associated with the pathogenesis of Alzheimer's disease," Molecular Medicine Reports, vol. 12, no. 3, pp. 4037-4042, 2015.

[68] Y. Zhang, M. Chopp, Y. Meng et al., "Effect of exosomes derived from multipluripotent mesenchymal stromal cells on functional recovery and neurovascular plasticity in rats after traumatic brain injury," Journal of Neurosurgery, vol. 122, no. 4, pp. 856-867, 2015.

[69] H. Xin, Y. Li, Y. Cui, J. J. Yang, Z. G. Zhang, and M. Chopp, "Systemic administration of exosomes released from mesenchymal stromal cells promote functional recovery and neurovascular plasticity after stroke in rats," Journal of Cerebral Blood Flow and Metabolism, vol. 33, no. 11, pp. 1711-1715, 2013.

[70] H. Xin, Y. Li, B. Buller et al., "Exosome-mediated transfer of miR-133b from multipotent mesenchymal stromal cells to neural cells contributes to neurite outgrowth," Stem Cells, vol. 30, no. 7, pp. 1556-1564, 2012.

[71] F. Agosta, D. Dalla Libera, E. G. Spinelli et al., "Myeloid microvesicles in cerebrospinal fluid are associated with myelin damage and neuronal loss in mild cognitive impairment and Alzheimer disease," Annals of Neurology, vol. 76, no. 6, pp. 813-825, 2014.

[72] A. D. Pusic and R. P. Kraig, "Youth and environmental enrichment generate serum exosomes containing miR-219 that promote CNS myelination," Glia, vol. 62, no. 2, pp. 284-299, 2014.

[73] Y. S. Takeda and Q. Xu, "Neuronal differentiation of human mesenchymal stem cells using exosomes derived from differentiating neuronal cells," PloS One, vol. 10, no. 8, article e0135111, 2015.

[74] S. A. Bellingham, B. M. Coleman, and A. F. Hill, "Small RNA deep sequencing reveals a distinct miRNA signature released in exosomes from prion-infected neuronal cells," Nucleic Acids Research, vol. 40, no. 21, pp. 10937-10949, 2012.

[75] R. F. Haseloff, S. Dithmer, L. Winkler, H. Wolburg, and I. E. Blasig, "Transmembrane proteins of the tight junctions at the blood-brain barrier: structural and functional aspects," Seminars in Cell \& Developmental Biology, vol. 38, pp. 16-25, 2015.

[76] B. D. Zipser, C. E. Johanson, L. Gonzalez et al., "Microvascular injury and blood-brain barrier leakage in Alzheimer's disease," Neurobiology of Aging, vol. 28, no. 7, pp. 977-986, 2007.

[77] A. Montecalvo, A. T. Larregina, and A. E. Morelli, "Methods of analysis of dendritic cell-derived exosome-shuttle microRNA and its horizontal propagation between dendritic cells," Methods in Molecular Biology, vol. 1024, pp. 19-40, 2013.

[78] A. S. Haqqani, C. E. Delaney, T. L. Tremblay, C. Sodja, J. K. Sandhu, and D. B. Stanimirovic, "Method for isolation and molecular characterization of extracellular microvesicles released from brain endothelial cells," Fluids and Barriers of the CNS, vol. 10, no. 1, p. 4, 2013.

[79] M. G. Banigan, P. F. Kao, J. A. Kozubek et al., "Differential expression of exosomal microRNAs in prefrontal cortices of schizophrenia and bipolar disorder patients," PloS One, vol. 8, no. 1, article e48814, 2013.

[80] G. Wang, B. C. Kwan, F. M. Lai, K. M. Chow, P. K. Li, and C. C. Szeto, "Urinary miR-21, miR-29, and miR-93: novel biomarkers of fibrosis," American Journal of Nephrology, vol. 36, no. 5, pp. 412-418, 2012.

[81] P. N. Alexandrov, P. Dua, J. M. Hill, S. Bhattacharjee, Y. Zhao, and W. J. Lukiw, "microRNA (miRNA) speciation in Alzheimer's disease (AD) cerebrospinal fluid (CSF) and extracellular fluid (ECF)," International Journal of Biochemistry and Molecular Biology, vol. 3, no. 4, pp. 365-373, 2012.

[82] L. Cheng, C. Y. Quek, X. Sun, S. A. Bellingham, and A. F. Hill, "The detection of microRNA associated with Alzheimer's disease in biological fluids using next-generation sequencing technologies," Frontiers in Genetics, vol. 4, p. 150, 2013.

[83] A. Tan, J. Rajadas, and A. M. Seifalian, "Exosomes as nanotheranostic delivery platforms for gene therapy," Advanced Drug Delivery Reviews, vol. 65, no. 3, pp. 357-367, 2013.

[84] K. B. Ebrahimi, N. Fijalkowski, M. Cano, and J. T. Handa, "Oxidized low-density-lipoprotein-induced injury in retinal pigment epithelium alters expression of the membrane complement regulatory factors CD46 and CD59 through exosomal and apoptotic bleb release," Advances in Experimental Medicine and Biology, vol. 801, pp. 259-265, 2014.

[85] J. J. Unternaehrer, A. Chow, M. Pypaert, K. Inaba, and I. Mellman, "The tetraspanin CD9 mediates lateral association of MHC class II molecules on the dendritic cell surface," Proceedings of the National Academy of Sciences of the United States of America, vol. 104, no. 1, pp. 234-239, 2007.

[86] K. Vartanian and M. Stenzel-Poore, "Toll-like receptor tolerance as a mechanism for neuroprotection," Translational Stroke Research, vol. 1, no. 4, pp. 252-260, 2010. 

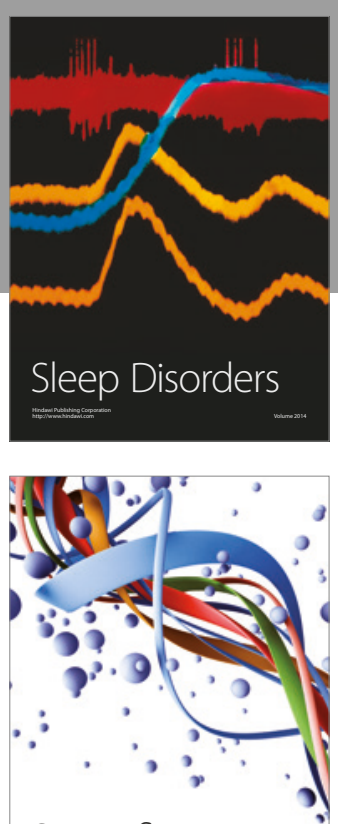

Scientifica
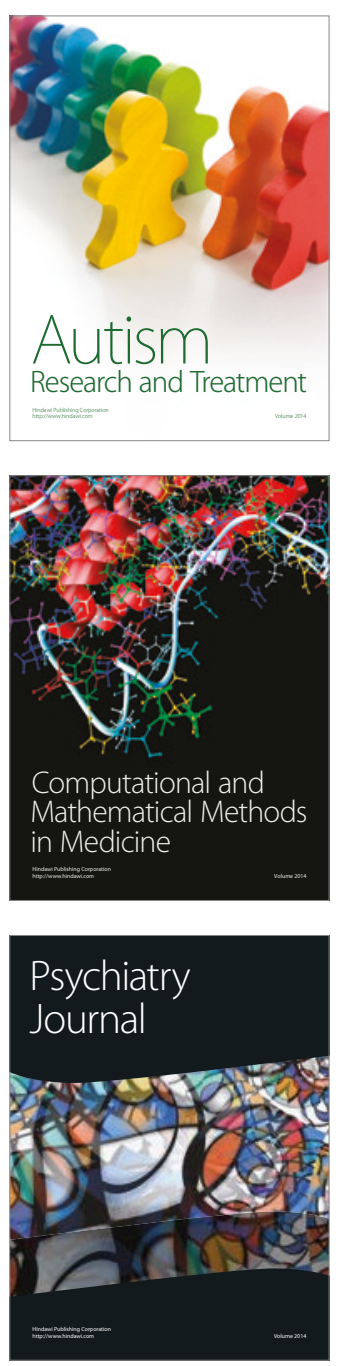
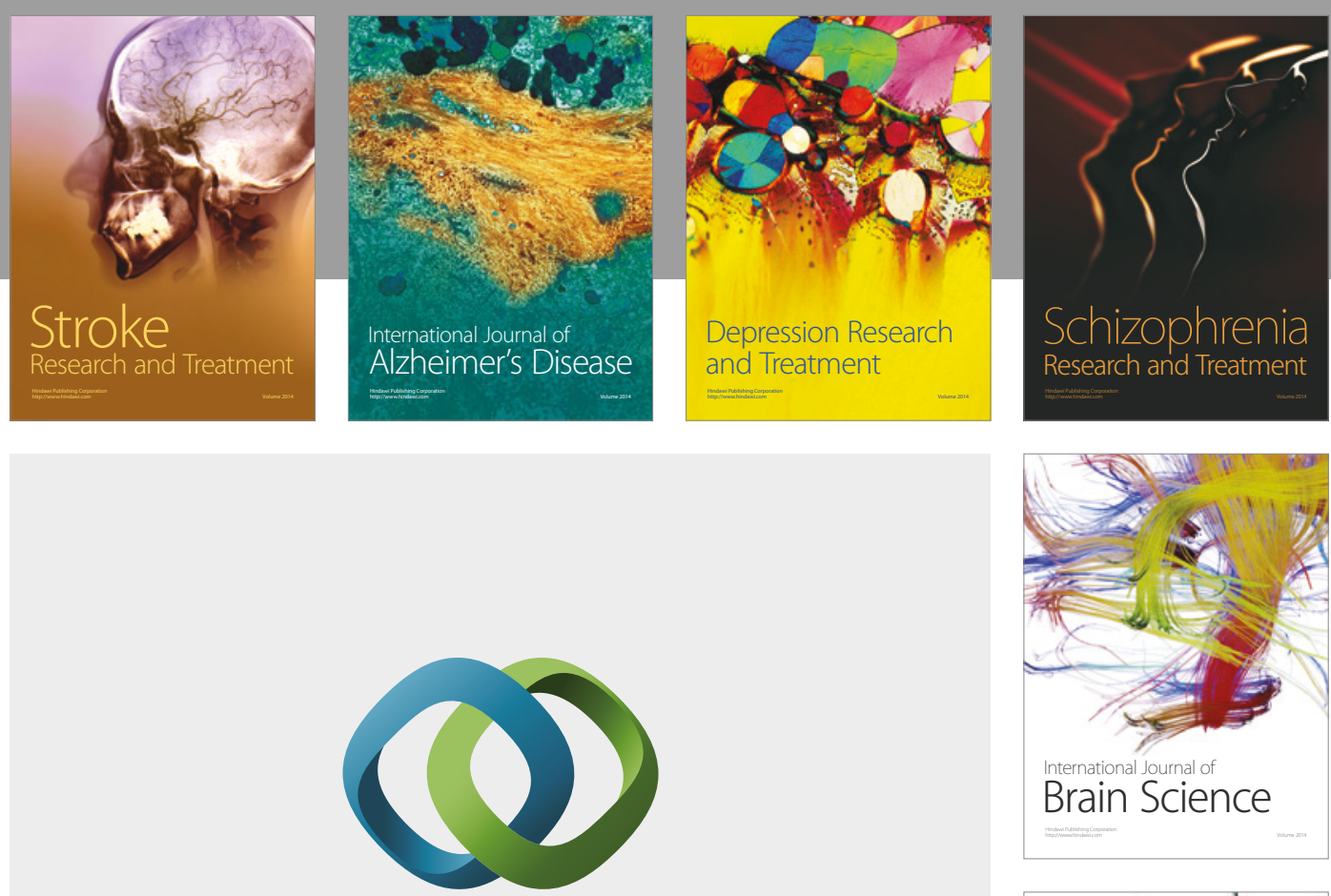

\section{Hindawi}

Submit your manuscripts at

https://www.hindawi.com
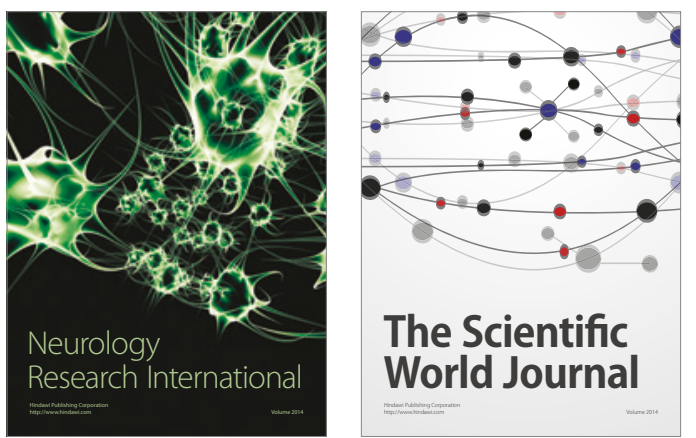

The Scientific World Journal

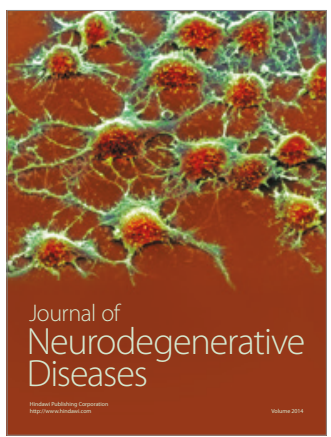

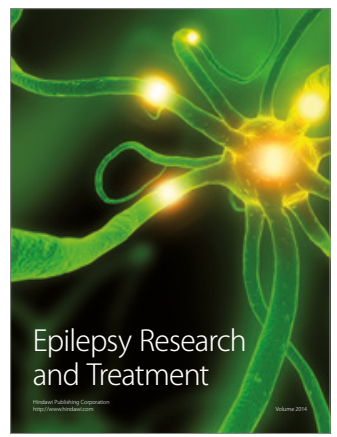

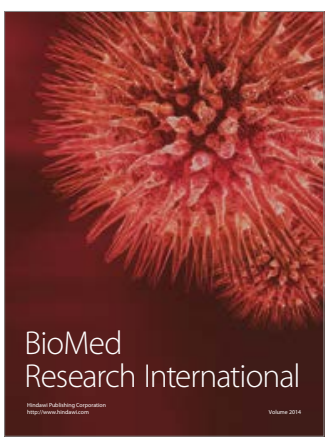

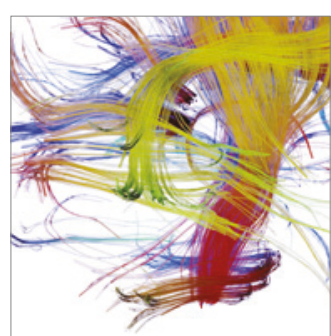

Brain Science

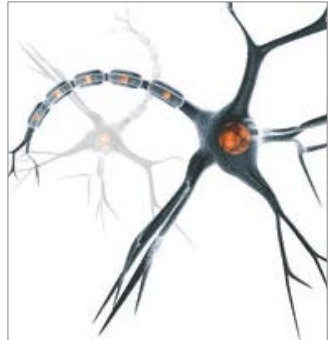

Neural Plasticity
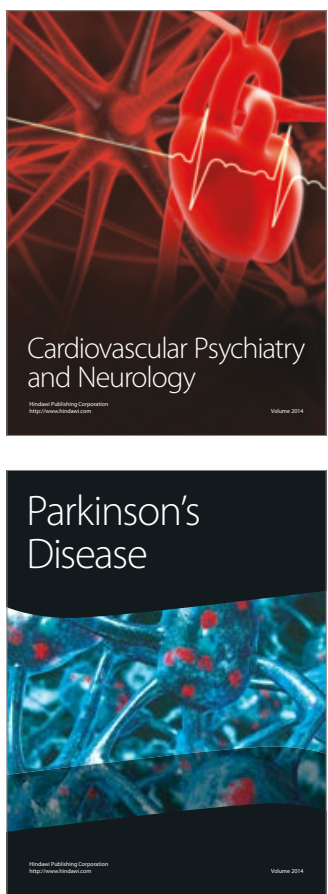